\title{
Effects on Road Safety and Functionality of Installing Countdown Timers to Traffic Lights
}

\author{
George Botzoris ${ }^{1}$, Nikolaos Papakatsikas ${ }^{1}$, Vassilios Profillidis ${ }^{1}$ and \\ Athanasios Galanis ${ }^{2}$ \\ ${ }^{1}$ Department of Civil Engineering, Democritus Thrace University, \\ Vas. Sofias 12, 67100, Xanthi, Greece \\ ${ }^{2}$ Department of Civil Engineering, University of Thessaly, \\ Pedion Areos 38334, Volos, Greece \\ gbotzori@civil.duth.gr,nikipapa@civil.duth.gr,bprofil@otenet.gr, \\ atgalanis@uth.gr
}

\begin{abstract}
In this paper it is surveyed whether the installation of countdown timers to the traffic lights of an urban intersection can efficiently contribute to the increase of both road safety and traffic capacity of the converging streets. A complete case-study is conducted in the city of Komotini (67,000 inhabitants, north-eastern Greece). By using video monitoring devices and the software Captiv L2100 the behavior of 14,946 drivers in two intersections in the center of the city under study is monitored. The analysis focused on their behavior just before the change of the traffic lights to green and red ones. It is concluded that installation of such devices leads to reduction of delays and increase of traffic capacity. However, concerning safety, drivers have a contradictory behavior. They respect the coming of the red light, while they begin moving before the green light is on. Under these findings, it is suggested to use the specific device before the light turns to red, but not before the light turns to green.
\end{abstract}

Keywords: road safety, traffic lights, countdown timers, delays, capacity, driver, behaviour

\section{Introduction}

Traffic signals with countdown timers were designed to inform drivers and pedestrians on the time remaining until the change of the current signal. They consist of two parts; a typical traffic light with the green, yellow (amber) and red signal heads and an additional head usually displaying a two-digit number that works as a countdown timer.

The countdown technology has been used for many years in pedestrian traffic lights with very positive results [1]. However, concerning driver traffic lights, timers are widespread mostly in East Asia, rather than in Europe or America.

Countdown timers can offer the following advantages:

- Improve the capacity of signalized intersections by reducing delay.

- Assist drivers to better understand traffic flows.

- Allow drivers to take decisions, while knowing the time disposal to act.

- Reduce accidents in intersections.

- Reduce anxiety before the start of a flow. Drivers will comfortably begin preparation, when they judge it is appropriate, given the time remaining until the green light. 
- Affect positively the environment as far as gas emissions and noise pollution are concerned by limiting the use of the acceleration pedal before starting.

The main safety issue countdown timers try to solve is red-light running. Knowing the time remaining until the change of the signal to green, the driver will, in theory, estimate more accurately whether he can cross the intersection safely or will need to decelerate and stop.

\section{Literature Review}

The Singapore Land Transport Authority in collaboration with the Nanyang Technological University installed a countdown timer to the traffic light of an intersection in 2003 [2]. During the research, which lasted one year, the number of vehicles that violated the red light was smaller for the first 4 months after installation to gradually return to initial percentages later. Concerning the drivers' behavior during the green light countdown, important accelerations were observed, resulting in an increase of the probability of rear shunts. The Singapore Land Transport Authority also judged that, since the country's road network consisted mainly of adaptive traffic lights, countdown timers would not be useful.

The previous research was repeated in 2006 at a different location in Singapore. Results revealed a reduction of $65 \%$ in red light violations 1.5 month after the installation, however, violations returned to previous levels after another 6 months. The number of drivers who decided to stop during the yellow light increased by 6.2 times one and a half month after the installation, a fact that remained unchanged afterwards [2].

Chen et al., (2007) studied 187 intersections in Taiwan between 2003 and 2006 and they noticed that in intersections where only the red signal was counted down, accidents and injuries decreased by 50\% [3]. On the other hand, in intersections where only the green signal was counted down, accidents doubled and injuries increased by $33 \%$. In intersections where the timer was used in both signals (green and red), an increase by $19 \%$ in collisions and $23 \%$ in injuries was measured.

Ibrahim et al., (2008) compared three intersections with timers to three intersections without timers in Malaysia and studied differences in driving behavior, vehicle headway and safety level [4]. Headway between two vehicles was defined as the time between the crossing of one vehicle and the crossing of the next one. Researchers did not remark any important difference in starting times, as delays were similar, especially for the first four vehicles of a queue. On the contrary, intersections with timers showed smaller headway values for all the vehicles in the queue, which implies that the saturation flow is quite higher than in other intersections. Concerning safety, the percentage of cycles where drivers violated the red light was measured slightly higher when timers were used; $30 \%$ against $24 \%$.

Limanond et al., (2009) concluded that in Thailand countdown timers reduced the average vehicle start up delay by roughly one (1) second, which slightly increased the intersection capacity [5]. The next year, Limanond et al., (2010) found that the installation of timers in Bangkok decreased the start up delay time by $22 \%$, there was no difference in drivers' behavior during the yellow signal and red light violations were reduced [6].

Sharma et al., (2009) analyzed queue discharge characteristics of two intersections, one with a timer and one without, in Chennai, India, through the use of a videotaping technique [7]. The traffic conditions in these roads are heterogeneous, as fast and slow vehicles share the same roadway and there is a lack of lane discipline. Results revealed that the timer clearly changed the headway distribution, substantially reducing the start-up lost time and increasing the discharge rate during the second half of the green phase.

Ma et al., (2010) in Shanghai found that the installation of countdown timers encouraged drivers to cross the intersection at higher speeds during the yellow phase, thus notably increasing the probability of collisions with other vehicles or pedestrians, mostly 
due to the speeding [8]. They also found that timers raised the intersection capacity and smoothed drivers' reactions to signal changes.

Chiou and Chang (2010) investigated the drivers' reactions to signal changes of traffic lights with countdown timers installed in Taipei [9]. They found a decrease in the percentage of drivers stopping too late during the green light countdown and an elongation of the dilemma zone (the distance from the vehicle stop line within which the driver's decision to stop or cross is not clear and either a rear shunt or a right-turn collision is possible) by about 28 meters. However, the percentage of drivers that start too early while waiting for the green light declined significantly and start up delays and vehicle headways were reduced as well. The positive effect on safety practically disappeared in a short time after the installation of timers; however the improvement in the capacity of the intersection remained even after four months.

Long et al., (2011) studied four intersections of similar traffic features in Changsha, central China, through videotaping; two intersections used countdown timers and two did not [10]. Their purpose was to investigate driver behavior mainly when the signal changed from green to yellow. Results showed that the possibility of violation of the red light almost doubled with timers installed, from $8.2 \%$ to $15.4 \%$. The crucial zone was the range of 10 to 30 meters away from the vehicle stop line. Without timers, vehicles crossed the stop line up until 4 seconds after the start of the yellow signal; therefore violations took place only during the first second of the red phase. Instead, vehicles in intersections with timers crossed even 7 seconds after the change to yellow, so violations occurred up to 4 seconds within the red phase. Generally, countdown timers did not seem to help drivers in decision-making or make their behavior safer.

Rijavec et al., (2013) showed that countdown timers in Ljubljana, Slovenia decrease red light violations, start up delays, while vehicle headways are not noticeably affected [11]. They also ran a survey among drivers which showed that $84 \%$ of them are positive to countdown timers, because they believe that they have the ability to prepare their actions in time. However, only $9 \%$ of the sample thought that safety was increased in the intersections.

Wenbo et al., (2013) studied two intersections (of four and five lanes per direction respectively) with high traffic flow and intense congestion in Guangzhou, south China, before and after the installation of timers [12]. They found that the roads' capacity increased by $10 \%$ at rush hours and by $5 \%$ at night. The extremely long signal cycles must be emphasized (120 and 170 seconds).

Papaioannou and Politis (2014) studied the changes in car drivers' behavior by comparing an intersection with countdown signal timers and a regular nearby one [13]. The intersections were located in the same city as those of this research. The paper focused on compliance of the drivers in two events; prior to the green phase and at the end of the green phase. $24 \%$ of the vehicles started early under a countdown timer, while the respective percentage for the other intersection was less than $1 \%$. It was also found that the drivers decide to cross on green phase termination $15 \%$ less when there is a countdown timer and generally took more correct decisions, either stopping or not.

Islam (2014) studied the safety and efficiency benefits of traffic signal countdown timers [14]. He performed an online survey which demonstrated an overall comprehension rate of $82 \%$, while $98 \%$ of the drivers believed the timers would help them in their decision-making process. Furthermore, with the use of a driving simulator, it was concluded that the countdown of the red signal resulted in the reduction of the first vehicle headway by 0.73 seconds or $22.5 \%$, while the countdown of the green signal increases drivers' stopping probability by $13.1 \%$.

It is easily perceived that research of the effects of installing countdown timers to traffic signals often reach results in conflict, which is mostly due to the different traffic circumstances of each case and the driving habits and social attitudes in the area. For example, some researchers found that the green signal countdown increases the 
probability of collisions, because drivers tend to focus on the timer and ignore traffic and other road events. Concerning the red signal countdown, research in Asia concluded that drivers are more careful of road events, as they don't need to constantly look at the traffic light, while European researchers state the opposite: drivers only mind the timer and ignore traffic in the intersection or other road users and pedestrians.

\section{Description of the Intersections and of the Data Recording Method}

\subsection{Description of the Intersections and the Traffic Lights}

Two intersections in the city centre of Komotini (67,000 inhabitants, north-eastern Greece) were selected under the criteria of high traffic flow, good visibility and relative similarity among them.

Traffic lights with countdown timers are used in the intersection of Dimosthenous Mpletsa and Apostolou Souzou streets (Figure 1). One of them controls traffic in Dimosthenous Mpletsa st. from the city centre to the suburbs and the other one controls traffic in Apostolou Souzou st. from west to east. Dimosthenous Mpletsa st. is 9.5m wide and two-way, with one lane in each direction, but only one direction was studied, for which no turns are allowed. Apostolou Souzou st. is $10 \mathrm{~m}$ wide and one-way, has two lanes and allows both right and left turns. There is also a signalized pedestrian crossing on this street. These two traffic lights were videotaped in spring 2013 during a workday, with the local market operating in the afternoon, under clear weather conditions. The shooting lasted 12 consecutive hours, from 08:00 to 20:00. From now on, the lights above will be called traffic light 1 and 2 respectively (2A is used for the left turns and $2 \mathrm{~B}$ for right turns and straight forward movement, Figure 1).

The traffic light without countdown timer is used on Dimokratias st., in the intersection with Orfeos, Thiseos and Dimosthenous Mpletsa streets (Figure 1). Dimokratias st. is $13 \mathrm{~m}$ wide and two-way, has two lanes per direction and a narrow median strip. We studied the direction towards Orfeos st., which is $6 \mathrm{~m}$ wide and vehicles move in three rows, along the existing two lanes. Only left turns are allowed. This light was videotaped in spring 2013 on a workday under clear weather conditions. The shooting lasted 12 consecutive hours, from 08:00 to 20:00. From now on, this light will be called traffic light 0 and will provide a benchmark for comparison, on which the results will be expressed.

The cycle of traffic light 0 lasts 80 seconds and the individual phases are 67 seconds red, 3 seconds yellow and 10 seconds green, while there is no separate left-turn phase. The cycle of traffic light 1 lasts 67 seconds and the phases are 34 seconds red, 3 seconds yellow and 30 seconds green. Traffic light 2 has a 67 second cycle which is divided into 49 seconds red, 3 seconds yellow and 15 seconds green, while there is no separate leftturn phase. 

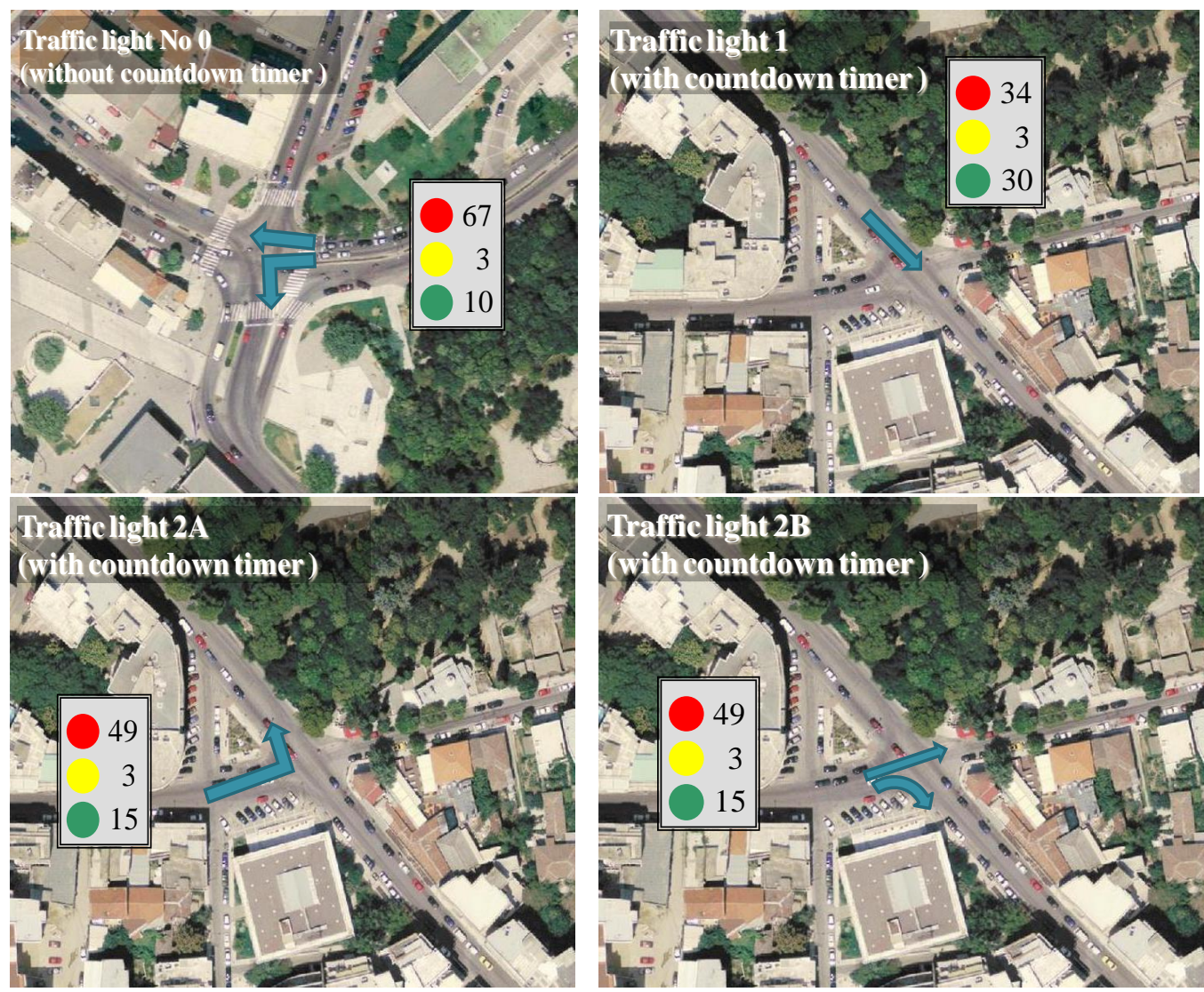

\section{Figure 1. Road Intersections and Installed Traffic Lights in the Study Area}

\subsection{Method of Data Acquisition and Analysis}

Captiv L2100 was used to analyze the video footage [15]. The software allows playing the video and simultaneously logging time events on user demand. It also allows for the time events to be categorized in description protocols, so that each of them is differentiated qualitatively. In practice, Captiv L2100 adds a database record for every vehicle crossing (when the user presses the specific button), which includes a time stamp and a code (different for crossing under red, yellow or green light). Next, using technical abilities of the software and MS Excel, the signal at the time of the crossing and the exact time within the cycle were defined for all vehicles.

\subsection{Road Traffic Data}

In total, the study included 14,946 vehicles which crossed the intersections. In particular, traffic light 0 was observed for 537 cycles, during which 3,533 vehicles crossed (Figure 2). Respectively, traffic light 1 was observed for 632 cycles, during which 5,538 vehicles crossed. The data analysis for traffic light 2 was divided into two parts; $2 \mathrm{~A}$, which included 3,581 vehicles in 583 cycles making a left turn and $2 \mathrm{~B}$, which included 2,294 vehicles in 584 cycles moving straight ahead or making a right turn. 

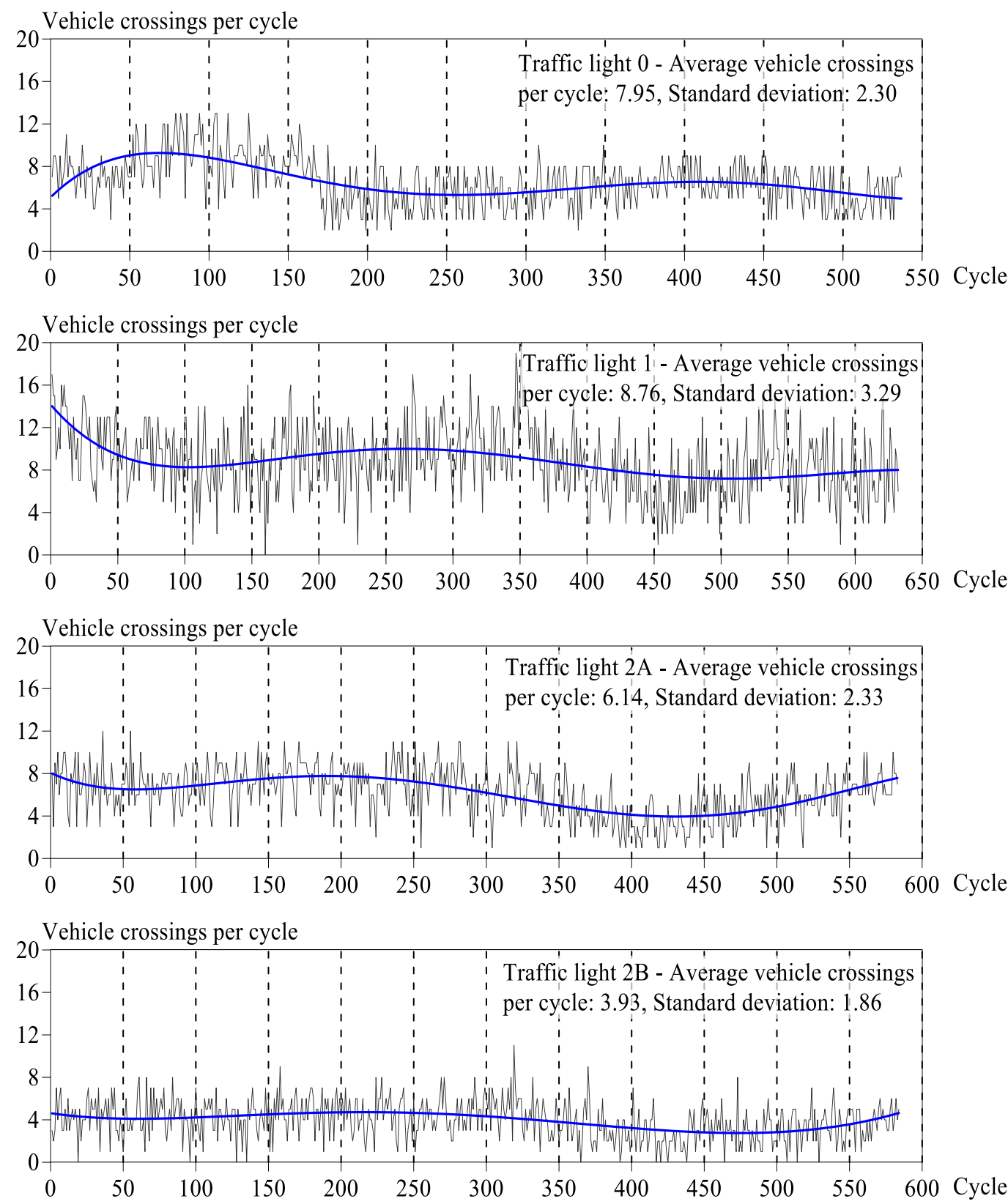

Figure 2. Traffic Flow Pattern of the Traffic Lights Under Study and Average Vehicle Crossings per Cycle

Initially, the number of vehicles that crossed in each cycle shows the similarity between the intersections as far as the pattern of the traffic volume during the day is concerned. Volumes are high in the beginning of the recording (roughly 08:00 in the morning), then gradually decline and increase again in the afternoon (13:00-14:00). Later in the afternoon, they are at the lowest point during the day, till the last hour of the recording, when they return to the afternoon levels (19:00-20:00). The above pattern is repeated for traffic lights $1,2 \mathrm{~A}$ and $2 \mathrm{~B}$, while 0 recorded a low number of vehicles in the evening hours.

The average number of crossing vehicles per cycle is lower in traffic light $2 \mathrm{~B}$, compared to the other road intersections, since Apostolou Souzou st. is a secondary street, hence the straight movement leads to a less important street, while right turns are served by the larger Dimosthenous Mpletsa st. On the other hand, left turns lead to the city 
centre, which justifies the higher volume. The generally high average ensures that there will be a sufficient number of cycles in which a waiting queue will be created before the vehicles start and/or more drivers will find themselves in the dilemma zone. Therefore, the traffic lights studied are satisfactory research points and the results can be considered as valid.

\section{Results}

\subsection{Violation of the Red Light because of Early Start or Late Crossing}

Red-light violations may present either during the start-up when a driver decides to cross the intersection before the change of signal to green, or before the signal change from yellow to red, should a driver not manage to respect light indicators. In this study, both cases were considered.

For the first case, the first vehicle of each cycle was defined and checked on whether it was waiting when the cycle started (beginning of the green phase). In order to ignore vehicles that crossed without stopping, since there were no vehicles waiting at the start of the cycle, only vehicles that crossed within 4 seconds after the signal change were considered. This ensured that the overwhelming majority of the recorded vehicles will be the first vehicles of a queue waiting for the light to change to green. Figure 3 shows the percentage of vehicles that crossed after the traffic signal changes from red to green (legal crossing) and of those that crossed before that moment (early start and illegal crossing).
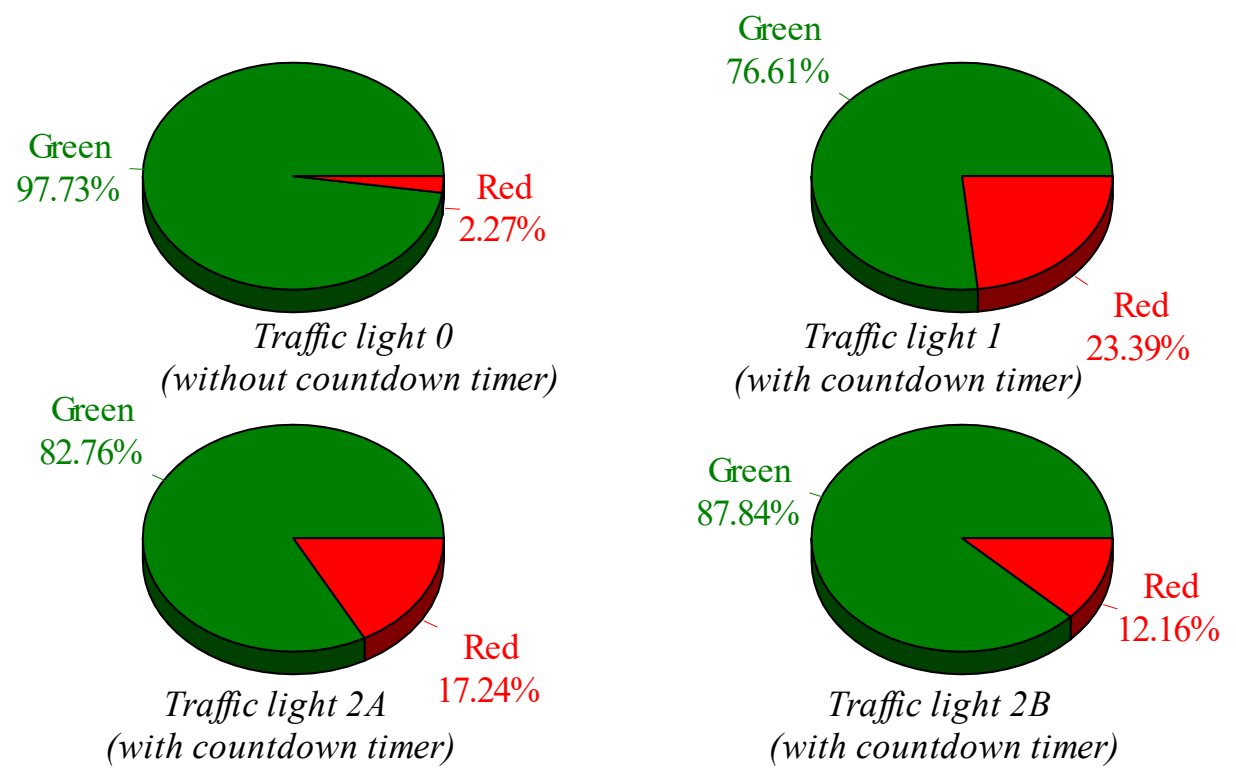

\section{Figure 3. Traffic Light Display when the Front Wheels of the First Vehicle of each Cycle Cross the Stop Line}

The above results are in line with previous research, [10], which indicate an important problem of countdown timers. While using traditional traffic signals (traffic light 0 ), very few vehicles under special circumstances decided to enter the intersection before the change of the signal to green, the countdown timer changes such a driving behavior, by influencing negatively safety. Most of the violations were committed by motorcycles and light vehicles. There were also occasions when the drivers did not stop at all before a red light, but continued moving at low speed, checked the intersection and crossed it 1 to 2 seconds before the start of the green phase. 
Furthermore, the second case of red-light violations was studied; crossing after the end of the yellow phase and the start of the red phase. In theory, available information about the remaining time to safely cross should reduce the number of violations and solve the problem for most drivers in the dilemma zone. Vehicles that crossed the last in a cycle were isolated and the percentage of them that crossed during the green, yellow or red signal was found (Figure 4). It must be noted that traffic light 0 "encourages" drivers to adopt a more dangerous approach because of the nature of its cycle, thus crossing regularly during the yellow phase and at times during the red phase.

From Figure 4, it is obvious that countdown timers do not potently affect this kind of violation. Illegal crossings may be clearly fewer, but that could be due to the difference in traffic light cycles. In any case, the percentage of illegal crossings remains high and in combination with the early starting (Figure 3) put the timers' effectiveness in reducing red light violations into question.
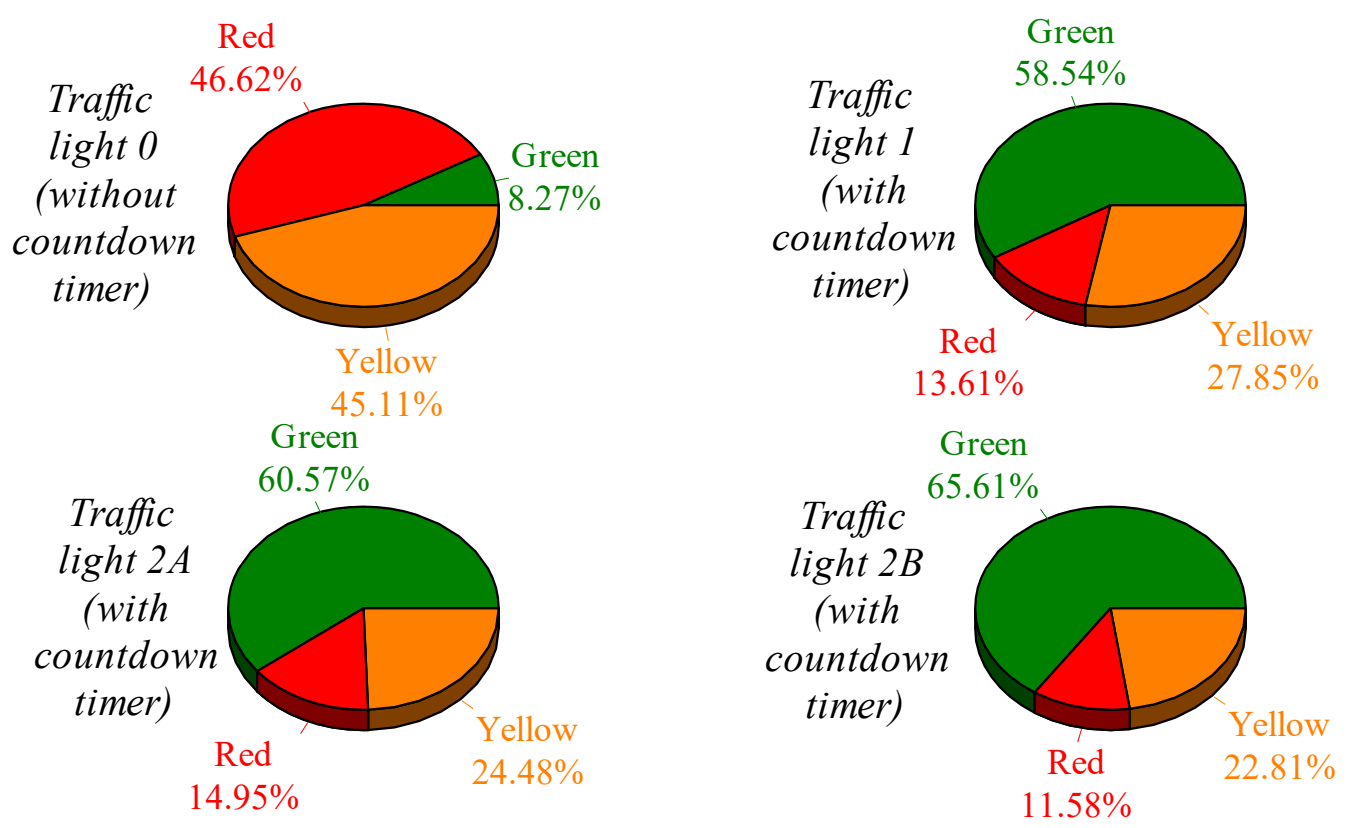

\section{Figure 4. Traffic Light Display when the Front Wheels of the Last Vehicle of each Cycle Cross the Stop Line}

This can also be inferred by the time stamps of the illegal crossings. Figure 5 illustrates the specific times when vehicles are crossing improperly, thus violating the existing light in a cycle (the point ordinates are random). Despite differences in percentages, qualitatively, the violation features are similar, as most illegal crossings occur during the first two seconds after the start of the red phase. An independent-samples t-test was conducted to compare the crossing time, after signal changes from yellow to red, of the red light violations with and without signal countdown timers. Indeed, there was a non significant difference in the scores with (mean: 1.014, std. dev.: 0.814) and without (mean: 0.824, std. dev.: 0.719) signal countdown timers; $\mathrm{t}(430)=-2.509, \mathrm{p}=0.112$. 

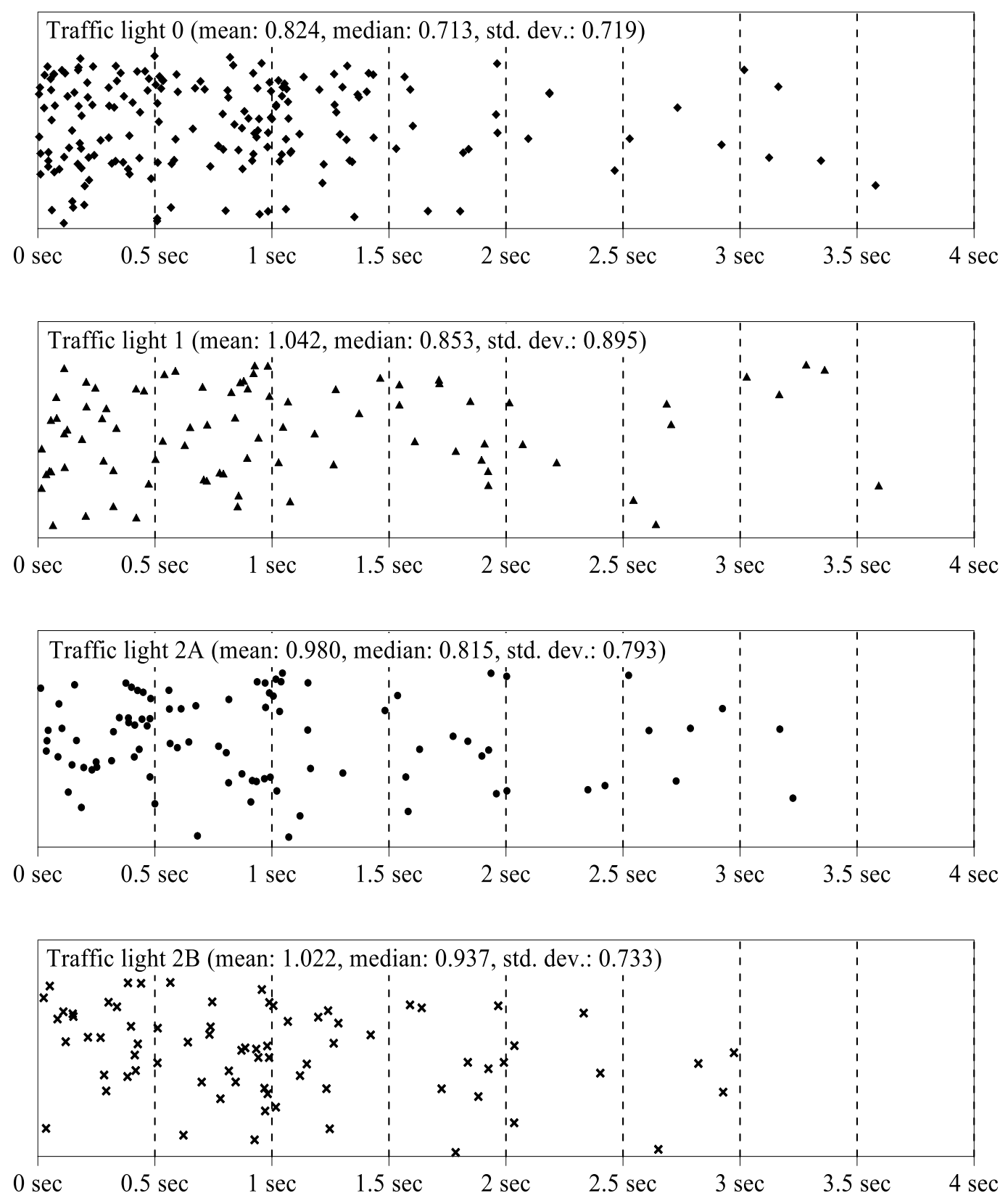

\section{Figure 5. Illegal Crossings of the Last Vehicles in each Cycle (each Point Indicates the Crossing Time, after Signal change to Red, of the Stop Line by the Front Wheels of the Last Vehicle of each Cycle)}

\subsection{Start up Delays}

The mainly negative effect in road safety seemingly caused by the countdown timers cannot be overlooked or counterbalanced by possible positive outcomes in road functionality and flow service. However, the study of the vehicles start-up delay times is interesting. The record analysis, apart from informing on whether each vehicle committed a violation, provided also the exact moment within the green phase when each vehicle crossed the intersection. Thus, Figure 6 was plotted, in which the crossing time of the first vehicles in a queue are noted, provided that they crossed at most 4 seconds after the change to green signal (the point ordinates are random).

It can be seen (Table 1 and Figure 6, next page) that in the traditionally signalized intersection (traffic light 0 ), no legal crossings occurred before at least one second after 
the start of the green signal. This is a result of the drivers' reaction time which causes the de facto shortening of the green phase by this amount of time. Instead, countdown timers in traffic lights $1,2 \mathrm{~A}$ and $2 \mathrm{~B}$ diminish the reaction time and legal crossings also occur during the first second of the green phase. It can be seen that during the first second $23.76 \%$ (traffic light 1), 27.8\% (traffic light 2A) and 21.25\% (traffic light 2B) of the drivers cross the stop line of the intersection. Consequently, countdown timers serve the traffic flows more efficiently by drastically reducing delays because of drivers being unprepared at start-up.

An independent-samples t-test was conducted to compare the first headway of each green phase with and without signal countdown timers. There was a significant difference in the scores with (mean: 1.619, std. dev.: 0.856) and without (mean: 2.114, std. dev.: 0.554 ) signal countdown timers; $\mathrm{t}(1424)=15.060, \mathrm{p}=0.000$. As first headway, is defined the time elapsed between the changing of the signal (from red to green) and the moment when the rear wheels of the first vehicle of each cycle cross the stop line [16, 17].

Table 1. Mean First (non-violating) Vehicle Headway (in sec) and Percentage (\%) of Vehicles Crossing the Stop Line in Specific Time Intervals

\begin{tabular}{lcccccc} 
& Mean first vehicle & \multicolumn{5}{c}{ Percentage (\%) of vehicles crossing } \\
& in the specific time intervals ( $\mathrm{t})$ & \\
& & $0 \leq \mathrm{t} \leq 0.5$ & $0.5<\mathrm{t} \leq 1$ & $1<\mathrm{t} \leq 2$ & $2<\mathrm{t} \leq 3$ & $\mathrm{t}>3$ \\
\hline Traffic light 0 & 2.114 & $0 \%$ & $0 \%$ & $49.33 \%$ & $42.29 \%$ & $8.38 \%$ \\
Traffic light 1 & 1.620 & $9.92 \%$ & $13.84 \%$ & $44.01 \%$ & $25.83 \%$ & $6.40 \%$ \\
Traffic light 2A & 1.551 & $12.86 \%$ & $14.94 \%$ & $42.74 \%$ & $23.44 \%$ & $6.02 \%$ \\
Traffic light 2B & 1.684 & $7.80 \%$ & $13.45 \%$ & $47.76 \%$ & $23.39 \%$ & $7.60 \%$ \\
\hline
\end{tabular}

\section{Conclusions - Proposal for further research}

The present research aimed at a comparative evaluation of driving behavior in intersections using traffic lights with countdown timers. The main advantage of the timers' installation seemed to be confirmed, as vehicle start-up delays were significantly reduced. However, the particular technology neither seems to assist drivers in better understanding the current traffic conditions, nor contributes to a decrease in violation frequency and consequently in the possibility of accidents. An important part of the responsibility should be allocated to drivers, since they use timers mostly to cross faster, rather than safer. The positive results in functionality cannot of course counterbalance the significant problems that appear concerning safety. 

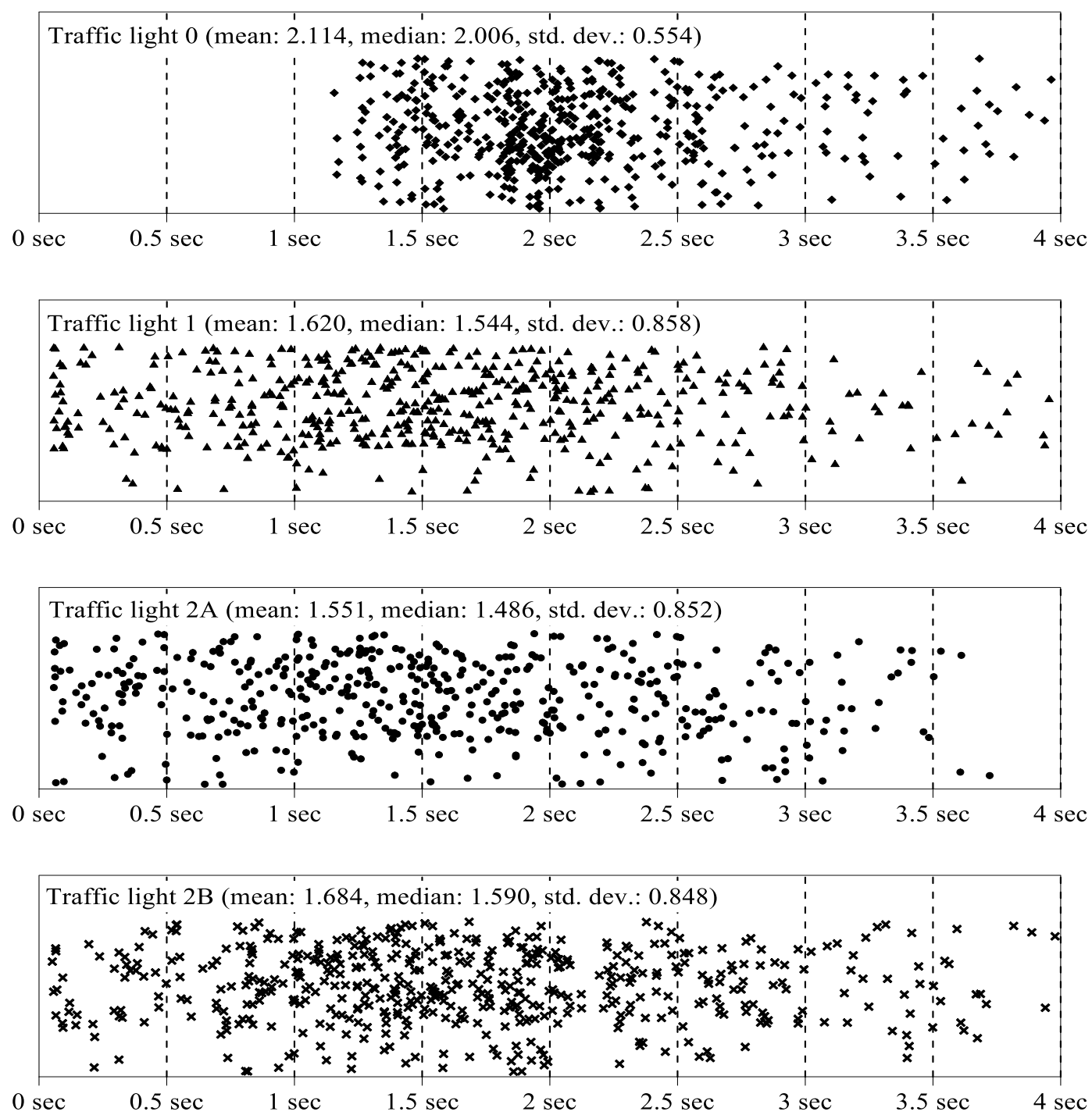

Figure 6. Legal Crossings of the First Vehicles in each Cycle (each Point indicates the Crossing Time, after Signal Change to Green, of the Stop Line by the Rear Wheels of the First Vehicle of each Cycle)

Apart from the obvious conclusion that timers, if installed, should exist in all legs of an intersection, as the partial installation emerges functionality and safety issues [3], the transportation engineer should also study driving habits in the particular area and examine one or more of the following solutions:

- Increase the all-red phase in the intersection. This may possibly reduce the possibility that the early start-up in one direction would be combined with a redlight crossing in the perpendicular direction. Consequently, the gained time in start-up at the beginning of the green phase is lost, but the drivers keep being less anxious when waiting for the signal change.

- Countdown timers stop showing the remaining time in the last seconds of the red phase. If after the indication that the red signal will change after 10 seconds, the timer goes out, drivers may begin their preparation for a well-timed start-up, but probably will not attempt to cross before the green signal, as they would not obtain more information than at a regularly signalized intersection. There lies the 
danger of estimation of the remaining time intuitively, which however would be a risky technique. In additionally, interrupting the provision of information could cause uncertainty to road users. In such a case, the gained start-up time will be smaller, though notable, by violations may well decline.

- Raise the length of the yellow phase and simultaneously decrease that of the green one. Analysis showed that timers lightly affect violations after the yellow signal changes to red. By increasing the yellow phase length, more time is given to drivers to assess the provided information, understand it and act accordingly. Possibly, some very defensive drivers may stop unnecessarily, however the gains in safety will be more important.

If even after the implementation of the above mentioned suggestions timers keep causing safety issues, they should be totally removed. In addition, the vast majority of these systems are not compatible with modern technology that allows for traffic-adaptive signaling and dynamic signal timing.

Future research may include study of the problem, dealt in the present paper, without countdown timers to traffic lights. In this way, a comparison of behavior of drivers in the cases of traffic lights with and without countdown timers could be concluded.

\section{Acknowledgments}

This paper is a revised and expanded version of a paper (in Greek) entitled "Effects on Safety by Installing Countdown Timers to Traffic Lights" presented at the $6^{\text {th }}$ Greek Road Safety Conference, Athens, March 12-13, 2015.

\section{References}

[1] P. Labrianidou, "Evaluation of Pedestrian Countdown Signal Timers at Signalized Intersections. The Case of Intersection Aigaiou - Andrianoupoleos in Kalamaria", M.Sc. Thesis, Postgraduate Programme: Planning, Organization and Management of Transport Systems, Aristotle University of Thessaloniki, Greece, (2010).

[2] K. M. Lum and H. Halim, "A Before-and-After Study on Green Signal Countdown Device Installation”, Transportation Research Part F: Traffic Psychology and Behavior, vol. 9, no. 1, (2006), pp. 29-41.

[3] H. Chen, H. Zhou and P. Hsu, "What do we Know about Signal Countdown Timers?", Institute of Transportation Engineers Journal, vol. 79, no. 7, (2009), pp. 72-76.

[4] M. R. Ibrahim, M. R. Karim and F. A. Kidwai, "The Effect of Digital Countdown Display on Signalized Junction Performance", American Journal of Applied Sciences, vol. 5, no. 5, (2008), pp. 479-482.

[5] T. Limanond, S. Chookerd and N. Roubtonglang, "Effects of Countdown Timers on Queue Discharge Characteristics of Through Movement at a Signalized Intersection", Transportation Research Part C: Emerging Technologies, vol. 17, no. 6, (2009), pp. 662-671.

[6] T. Limanond, P. Prabjabok and K. Tippayawong, "Exploring Impacts of Countdown Timers on Traffic Operations and Driver Behavior at a Signalized Intersection in Bangkok", Transport Policy, vol. 17, no. 6, (2010), pp. 420-427.

[7] A. Sharma, L. Vanajakshi and N. Rao, "Effect of Phase Countdown Timers on Queue Discharge Characteristics under Heterogeneous Traffic Conditions", Journal of the Transportation Research Board, vol. 2130, (2009), pp. 93-100.

[8] W. Ma, Y. Liu and X. Yang, "Investigating the Impacts of Green Signal Countdown Devices: Empirical Approach and Case Study in China", Journal of Transportation Engineering, vol. 136, no. 11, (2010), pp. 1049-1055.

[9] Y. C. Chiou and C. H. Chang, "Driver Responses to Green and Red Vehicular Signal Countdown Displays: Safety and Efficiency Aspects", Accident Analysis and Prevention, vol. 42, no. 4, (2010), pp. 1057-1065.

[10] K. Long, L. Han and Q. Yang, "Effects of Countdown Timers on Driver Behavior after the Yellow Onset at Chinese Intersections”, Traffic Injury Prevention, vol. 12, no. 5, (2011), pp. 38-544.

[11] R. Rijavec, J. Zakovšek and T. Maher, "Acceptability of Countdown Signals at an Urban Signalized Intersection and their Influence on Drivers Behaviour", Promet Traffic and Transportation, vol. 25, no. 1, (2013), pp. 63-71. 
[12] S. Wenbo, H. Zhaocheng, X. Xi and X. Feifei, "Exploring Impacts of Countdown Timers on Queue Discharge Characteristics of Through Movement at Signalized Intersections", Procedia - Social and Behavioral Sciences, vol. 96, no. 6, (2013), pp. 255-264.

[13] P. Papaioannou and I. Politis, "Preliminary Impact Analysis of Countdown Signal Timer Installations at two Intersections in Greece", Procedia Engineering, vol. 84, (2014), pp. 634-647.

[14] M. R. Islam, "Safety and Efficiency Benefits of Traffic Signal Countdown Timers: A Driving Simulator Study", Ph.D. Thesis, Oregon State University, Corvallis (OR), USA, (2014).

[15] Technology Ergonomics Applications, "CAPTIV L2100 - Software for Task Analysis", Retrieved from: http://teaergo.com/site/en/products/manufacturers/tea/captiv-software/captiv-12100,

(2016) February 26.

[16] P. Dey, S. Nandal and R. Kalyan, "Queue Discharge Characteristics at Signalised Intersections under Mixed Traffic Conditions”, European Transport, no. 55, (2013), pp. 1-21.

[17] C. J. Bester and W. L. Meyers, "Saturation Flow Rates", Proceedings of the 26th Southern African Transport Conference (SATC 2007), Pretoria, South Africa, (2007) July 9-12, pp. 560-568.

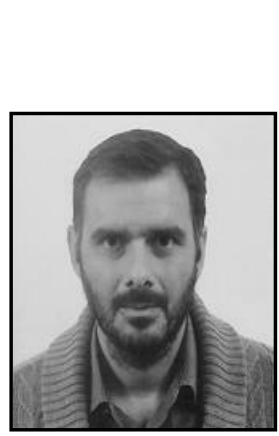

\section{Authors}

George Botzoris is Assistant Professor at the Section of Transportation of Democritus University of Thrace, where he has been teaching since 2008. He has the Diploma of Civil Engineering, M.Sc. in Business Administration and Ph.D. in Transportation. His research interests include transport economics, public transport planning, travel behavior analysis and modeling, analysis and forecast of demand, sustainable mobility and effects of transport activities on the environment. He has written to this day over 100 scientific papers that have been published in scientific journals as well as conference proceedings.

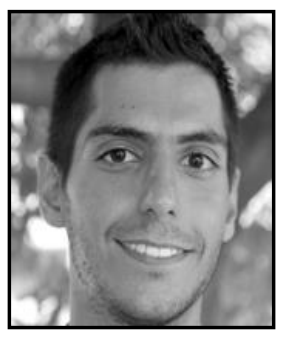

Nikolaos Papakatsikas is Research Associate at the Section of Transportation of Democritus University of Thrace. He has the Diploma of Civil Engineering. He has focused on transportation engineering and planning, emphasizing in assessing the performance of various intelligent systems and traffic control methods. He is also interested in the application of mathematics and statistics on traffic conditions, as well as the development of innovative technology aiming to improve urban transportation efficiency and experience.

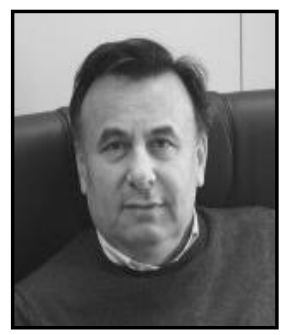

Vassilios Profillidis is Professor at the Section of Transportation of Democritus University of Thrace, where he has been teaching since 1990. He has the Diploma of Civil Engineering of the Univ. of Thessaloniki, DEA and Ph.D. in Transportation of the Ecole Nationale des Ponts et Chaussees of Paris and the Diploma of Law of the Univ. of Thessaloniki. He has been an advisor to many transport authorities. He has taken part in many international conferences as well as meetings of the European Union, the World Bank and the European Conference of Ministers of Transport (ECMT). He has carried out a number of transportation studies, traffic studies, feasibility studies, organization and management studies, airport master plans, and metro and railway track designs. He has written to this day 9 books and over 130 scientific papers that have been published in scientific journals as well as conference proceedings. 


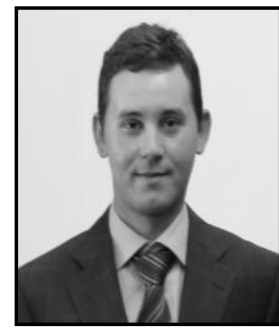

Dr Athanasios Galanis received his B.Sc. in Civil Engineering in 2006 and his Ph.D. in Transportation Engineering in 2011 from the Department of Civil Engineering, University of Thessaly, Greece. He also obtained his M.Sc. in Transportation Engineering in Aristotle University of Thessaloniki in 2007. His research topics are road design, road safety and sustainable transportation. He has written to this day over 50 scientific papers that have been published in scientific journals as well as conference proceedings. 